\title{
REMARKS ON A MONOTONE MARKOV CHAIN ${ }^{*}$
}

P. Todorovic

Statistics and Applied Probability Program

University of California, Santa Barbara

\begin{abstract}
In applications, considerations on stochastic models often involve a Markov chain $\left\{\zeta_{n}\right\}_{0}^{\infty}$ with state space in $R_{+}$, and a transition probability $\mathrm{Q}$. For each $\mathrm{x} \varepsilon \mathrm{R}_{+}$the support of $\mathrm{Q}(\mathrm{x}$, .) is $[0, \mathrm{x}]$. This implies that $\zeta_{0} \geq \zeta_{1} \geq \ldots$. Under certain regularity assumptions on $\mathrm{Q}$ we show that $\mathrm{Q}^{\mathrm{n}}\left(\mathrm{x}, \mathrm{B}_{\mathrm{u}}\right) \rightarrow 1$ as $\mathrm{n} \rightarrow \infty$ for all $\mathrm{u}>0$ and that $1-Q^{n}\left(x, B_{u}\right) \leq\left[1-Q\left(x, B_{u}\right)\right]^{n}$ where $B_{u}=[0, u)$. Set $\tau_{0}=$ $\max \left\{\mathrm{k} ; \zeta_{\mathrm{k}}=\zeta_{0}\right\}, \tau_{\mathrm{n}}=\max \left\{\mathrm{k} ; \zeta_{\mathrm{k}}=\zeta_{\tau_{\mathrm{n}-1}+1}\right\}$ and write $\mathrm{X}_{\mathrm{n}}=\zeta_{\tau_{\mathrm{n}-1+1}}$, $\mathrm{T}_{\mathrm{n}}=\tau_{\mathrm{n}}-\tau_{\mathrm{n}-1}$. We investigate some properties of the imbedded Markov chain $\left\{X_{n}\right\}_{0}^{\infty}$ and of $\left\{T_{n}\right\}_{0}^{\infty}$. We determine all the marginal distributions of $\left\{\mathrm{T}_{\mathrm{n}}\right\}_{0}^{\infty}$ and show that it is asymptotically stationary and that it possesses a monotonicity property. We also prove that under some mild regularity assumptions on $\beta(x)=1-Q\left(x, B_{x}\right)$,

$$
\sum_{1}^{n}\left(T_{i}-a\right) / b \sqrt{n} \stackrel{d}{\rightarrow} Z \sim N(0,1) .
$$
\end{abstract}

Key words and phrases: Markov chain, asymptotic stationarity, mixing property, central limit theorem.

AMS subject classifications: Primary 60 j05, Secondary 60k 30.

\section{INTRODUCTION}

Set $R_{+}=[0, \infty)$ and denote by $B\left(R_{+}\right)$the $\sigma$-field of Borel subsets in $R_{+}$. Various applications often involve a Markov chain $\left\{\zeta_{n}\right\}_{0}^{\infty}$ with state space in $R_{+}$, an initial distribution $\mu$ and a transition probability $\mathrm{Q}$. The $\mu$ and $\mathrm{Q}$ determine completely and uniquely a probability measure $\mathrm{P}_{\mu}$ on the measure space $\left\{\mathrm{R}_{+}^{\infty}, \mathcal{B}\left(\mathrm{R}_{+}^{\infty}\right)\right\}$. We shall

\footnotetext{
* Received 11/27/87, accepted 1/11/88.
} 
write $\mathrm{P}_{\mathrm{x}}$ instead of $\mathrm{P}_{\mu}$ when $\mu$ is the Dirac measure $\varepsilon_{\mathrm{x}}(\cdot)$ on $B\left(\mathrm{R}_{+}\right)$. Corresponding to the probability measure $\mathrm{P}_{\mu}$ is the expectation operator $\mathrm{E}_{\mu}$.

Throughout this paper we assume that $Q$ satisfies the following conditions:

$$
\begin{gathered}
\mathrm{A}_{1}: \forall \mathrm{x} \quad \in \mathrm{R}_{+} \text {the support of } \mathrm{Q}(\mathrm{x}, .) \text { is }[0, \mathrm{x}] \\
\mathrm{A}_{2} ; \forall \mathrm{x}>0
\end{gathered}
$$

$$
Q(x,\{y\})= \begin{cases}0 & x \neq y \\ \beta(x)>0 & x=y\end{cases}
$$

From $A_{1}$ and $A_{2}$ it follows at once that

$$
\zeta_{0} \geq \zeta_{1} \geq \ldots \text { and that } \mathrm{Q}(0,\{0\})=\beta(0)=1
$$

Set $B_{x}=[0, x)$ and $B_{x}^{\prime}=[0, x]$; in addition to $A_{1}$ and $A_{2}$ we also assume that:

$$
A_{3}: Q\left(x_{1},\left[y, x_{1}\right)\right) \leq Q\left(x_{2},\left[y, x_{2}\right)\right) \text { for all } 0 \leq y \leq x_{1} \leq x_{2}
$$

$\mathrm{A}_{4}$ : The chain is stochastically monotone, i.e., for any $0 \leq y \leq x_{1} \leq x_{2}$ ( see [4]).

$$
\mathrm{Q}\left(\mathrm{x}_{2}, \mathrm{~B}_{\mathrm{y}}\right) \leq \mathrm{Q}\left(\mathrm{x}_{1}, \mathrm{~B}_{\mathrm{y}}\right)
$$

From (1.1) it follows that $\mathrm{Q}\left(\mathrm{x},{ }^{\cdot}\right)$ is an atomic probability measure whose only atom is $\{x\}$.

Markov processes of this type arise in various applications. A particular problem which motivated consideration of $\left\{\zeta_{n}\right\}_{0}^{\infty}$ is related to modeling of the long term effect of close up surface soil erosion on crop production. It has been shown (Todorovic et al., 1987 and Todorovic and Gani., 1987) that the sequence of annual yields $\left\{\mathrm{X}_{\mathrm{n}}\right\}_{0}^{\infty}$ of a crop, from a crop growing area subject to soil erosion, is specified as

$$
\mathrm{X}_{\mathrm{n}}=\mathrm{Y}_{\mathrm{n}} \mathrm{L}_{\mathrm{n}} \text {. }
$$

Here $\left\{\mathrm{Y}_{\mathrm{n}}\right\}_{0}^{\infty}$ is an i.i.d. sequence of positive r.v.'s and $\left\{\mathrm{L}_{\mathrm{n}}\right\}_{0}^{\infty}$ a Markov process independent of $\left\{\mathrm{Y}_{\mathrm{n}}\right\}$ and such that $1 \equiv \mathrm{L}_{0} \geq \mathrm{L}_{1} \geq \mathrm{L}_{2} \geq$.. 
Let $\zeta_{0}$ be the depth of top soil of the crop growing area at time $t=0$; let $\zeta_{1}$ be its depth one year later, and so on. We clearly have that $\zeta_{0} \geq \zeta_{1} \geq \ldots$. One can also show that $L_{n}=\varphi\left(\zeta_{n}\right)$, where $\varphi: R_{+} \rightarrow[0,1]$ is strictly increasing, continuous on $\left[0, d_{0}\right)$ and such that $\varphi(0)=0$ and $\varphi(u)=1$ for $u \geq d_{0}$.

Due to the fact that $\zeta_{n}=\varphi^{-1}\left(L_{n}\right)$, it seems reasonable to assume that $\left\{\zeta_{n}\right\}_{0}^{\infty}$ represents a Markov process with transition probability $\mathrm{Q}$ satisfying regularity conditions $\mathrm{A}_{1}-\mathrm{A}_{4}$. The atom $\{\mathrm{x}\}$ of $\mathrm{Q}\left(\mathrm{x},{ }^{*}\right)$ reflects the fact that there may be no soil loss due to erosion in a particular year. On the other hand, the smaller depth of the top soil layer the larger is the probability of reaching a lower level $y$. This justifies the monotonicity assumption $\mathrm{A}_{4}$. Stochastic monotonicity is a very common phenomenon.

Random walks, diffusions, birth and death and branching processes have this property. Another problem where the process $\left\{\zeta_{n}\right\}_{0}^{\infty}$ can be used is to model the decrease of a dam's capacity due to silting.

Denote by $T_{0}$ the time the chain stays in its initial state. Let $T_{1}$ be the duration of its stay in the next state, and so on. We investigate a number of properties of the sequence $\left\{\mathrm{T}_{\mathrm{n}}\right\}_{0}^{\infty}$. We determine all its marginal distributions, and show that this process is asymptotically stationary and that it possesses a mixing property. We also show that under certain regularity assumptions on $\beta(\cdot)$

$$
\begin{aligned}
& \sum_{1}^{n}\left(T_{i}-a\right) / b \sqrt{n} \stackrel{d .}{\rightarrow} Z \sim N(0,1) \\
& \text { where } a=(1-p)^{-1} \text { and } b=a \sqrt{p}
\end{aligned}
$$

\section{SOME PROPERTIES OF $Q^{n}$}

Here we discuss some basic features of the n-step transition probability $Q^{\mathrm{n}}$ and of

$$
\beta(x)=1-Q\left(x, B_{x}\right)
$$

which follow from assumptions $A_{1}-A_{4}$. First of all, from $A_{3}$ we have

$$
\beta\left(x_{1}\right) \geq \beta\left(x_{2}\right) \text { for all } 0 \leq x_{1} \leq x_{2} \text {. }
$$


As usual, we define the $n$-step transition probabilities by setting $Q^{1}(x, B)=Q(x, B)$ and for $n \geq 2$

$$
Q^{n}(x, B)=\int Q(s, B) Q^{n-1}(x, d s)
$$

where $\mathrm{B} \in \mathrm{B}_{\mathrm{x}}^{\prime} \cap \mathcal{B}\left(\mathrm{R}_{+}\right)$. From this we clearly have:

$$
Q^{n}(x,\{x\})=(\beta(x))^{n}
$$

We now prove the following:

\section{Proposition 2.1}

For every $\mathrm{n}=1,2, \ldots$ and $\mathrm{u} \geq 0$

$$
1-Q^{n}\left(x, B_{u}^{\prime}\right) \leq\left[1-Q\left(x, B_{u}^{\prime}\right)\right]^{n}
$$

Proof:

Since

$$
\mathrm{P}_{\mathrm{x}}\left\{\zeta_{\mathrm{n}} \leq \mathrm{u}\right\}=\mathrm{P}_{\mathrm{x}}\left\{\zeta_{\mathrm{n}-1} \leq \mathrm{u}\right\}+\mathrm{P}_{\mathrm{x}}\left\{\zeta_{\mathrm{n}-1}>\mathrm{u}, \zeta_{\mathrm{n}} \leq \mathrm{u}\right\}
$$

we have that

$$
Q^{n}\left(x, B_{u}^{\prime}\right)=Q^{n-1}\left(x, B_{u}^{\prime}\right)+\int_{(u, x]} Q\left(y, B_{u}^{\prime}\right) Q^{n-1}(x, d y)
$$

This and (1.4) yield:

$$
Q^{n}\left(x, B_{u}^{\prime}\right) \geq Q^{n-1}\left(x, B_{u}^{\prime}\right)+Q\left(x, B_{u}^{\prime}\right)\left[1-Q^{n-1}\left(x, B_{u}^{\prime}\right)\right],
$$

from which we obtain the following recursion

$$
1-Q^{n}\left(x, B_{u}^{\prime}\right) \leq\left[1-Q^{n-1}\left(x, B_{u}^{\prime}\right)\right]\left[1-Q\left(x, B_{u}^{\prime}\right)\right],
$$

which proves (2.5).

Corollary 2.1

From (2.5) and (2.6) it follows that 


$$
\mathrm{Q}^{\mathrm{n}}\left(\mathrm{x}, \mathrm{B}_{\mathrm{u}}\right) \leq \mathrm{Q}^{\mathrm{n}+1}\left(\mathrm{x}, \mathrm{B}_{\mathrm{u}}\right) \text { and } \mathrm{Q}^{\mathrm{k}}\left(\mathrm{x}, \mathrm{B}_{\mathrm{u}}\right) \rightarrow 1
$$

as $\mathrm{k} \rightarrow \infty$. This and (1.2) imply that

$$
\zeta_{n} \rightarrow 0 \text { (a.e) } P_{x} \text {. }
$$

In addition, from (2.5) we also obtain that

$$
\mathrm{Q}^{\mathrm{n}}\left(\mathrm{x}, \mathrm{B}_{\mathrm{u}}^{\prime}\right) \geq 1-\exp \left\{-\mathrm{nQ}\left(\mathrm{x}, \mathrm{B}_{\mathrm{u}}^{\prime}\right\}\right.
$$

for all $\mathrm{n}=1,2, \ldots, \quad$ which gives some information concerning the rate of convergence of $\mathrm{Q}^{\mathrm{k}}\left(\mathrm{x}, \mathrm{B}_{\mathrm{u}}^{\prime}\right)$ to 1 .

Proposition 2.2

The function $\beta(x)$ is continuous at every $x>0$.

Proof:

From (1.4) and (2.1) we have:

$$
\mathrm{Q}\left(\mathrm{x}_{2}, \mathrm{~B}_{\mathrm{x}_{1}}\right) \leq 1-\beta\left(\mathrm{x}_{1}\right) .
$$

Hence, $\left(0<x_{1}<x_{2}\right)$

$$
\mathrm{Q}\left(\mathrm{x}_{2}, \mathrm{~B}_{\mathrm{x}_{2}}\right)-\mathrm{Q}\left(\mathrm{x}_{2}, \mathrm{~B}_{\mathrm{x}_{1}}\right) \geq \beta\left(\mathrm{x}_{1}\right)-\beta\left(\mathrm{x}_{2}\right) \geq 0 .
$$

Letting $\mathrm{x}_{1} \uparrow \mathrm{x}_{2}$ we obtain:

$$
\beta(x-0)-\beta(x)=0 \text { for all } x>0,
$$

which proves that $\beta(x)$ is left continuous.

On the other hand, according to (1.1), $Q(x,\{y\})=0$ for all $x>0$ and $0 \leq y<x$. Thus,

$$
\mathrm{Q}(\mathrm{y}+0,\{\mathrm{y}\})=0 \text {. }
$$

Now, from (2.1) and (2.2) we have for every $x>0$ and $\varepsilon>0$

$$
Q\left(x+\varepsilon, B_{x+\varepsilon}\right)-Q\left(x, B_{x}\right)=\beta(x)-\beta(x+\varepsilon) \geq 0
$$


By letting $\varepsilon \rightarrow 0$ we obtain:

$$
\begin{gathered}
Q\left(x+0, B_{x}^{\prime}\right)-Q\left(x, B_{x}\right)=\beta(x)-\beta(x+0) \geq 0 \\
Q(x+0,\{x\})+Q\left(x+0, B_{x}\right)-Q\left(x, B_{x}\right)=\beta(x)-\beta(x+0) \geq 0 .
\end{gathered}
$$

This, (1.4), and (2.10) prove the assertion.

\section{Remark 2.1}

From the last inequality and condition (1.4) we have

$$
Q\left(x+0, B_{x}\right)=Q\left(x, B_{x}\right) \text {. }
$$

Remark 2.2

Inequality (2.2) implies that the closer the chain is to the state $\{0\}$, the larger is the probability that the next step will lead to the same state. However, it seems reasonable to assume that regardless of how close the chain is to the zero state, the probability of landing somewhere in $[0, x)$, given that it was in $\mathrm{x}$, is a positive number. This gives rise to the following assumption:

$$
\beta(0+)=1-\lim _{x \rightarrow 0} Q\left(x, B_{x}\right)=p<1
$$

\section{AN IMBEDDED MARKOV CHAIN}

Set $\tau_{0}=\max \left\{\mathrm{k} ; \zeta_{\mathrm{k}}=\zeta_{0}\right\}, \tau_{\mathrm{n}}=\max \left\{\mathrm{k} ; \zeta_{\mathrm{k}}=\zeta_{\tau_{\mathrm{n}-1}+1}\right\}$ and write

$$
\mathrm{X}_{0}=\zeta_{0}, \quad \mathrm{X}_{\mathrm{n}}=\zeta_{\tau_{\mathrm{n}-1}+1}, \quad \mathrm{n}=1,2, \ldots
$$

Clearly $X_{0}>X_{1}>\ldots$ and $X_{n} \rightarrow 0$ (a.e) $P_{x}$. One can verify that $\left\{X_{n}\right\}_{0}^{\infty}$ is also a Markov chain with initial distribution $\mu$ and transition probability $\mathrm{P}$ defined as

$$
P(x, d y)=\left\{\begin{array}{cc}
\frac{Q(x, d y)}{1-\beta(x)} & x>0 \\
0 & x=0
\end{array}\right.
$$

The support of $\mathrm{P}\left(\mathrm{x},,^{*}\right)$ is clearly $[0, \mathrm{x})$. As in (2.7) we have 


$$
\mathrm{P}^{\mathrm{n}}\left(\mathrm{x}, \mathrm{B}_{\mathrm{u}}\right) \leq \mathrm{P}^{\mathrm{n}+1}\left(\mathrm{x}, \mathrm{B}_{\mathrm{u}}\right) \text { and } \mathrm{P}^{\mathrm{n}}\left(\mathrm{x}, \mathrm{B}_{\mathrm{u}}\right) \rightarrow \mathrm{P}_{0}\left(\mathrm{x}, \mathrm{B}_{\mathrm{u}}\right)=1 \text {, }
$$

where $\mathrm{P}^{\mathrm{n}}$ is the $\mathrm{n}$-step transition probability of the chain $\left\{\mathrm{X}_{\mathrm{n}}\right\}_{0}^{\infty}$.

The $\mathrm{P}^{\mathrm{n}}$ can be interpreted as a non-negative linear operator on the cone of nonnegative Borel functions by defining:

$$
\left(P^{n} h\right)(x)=\int_{[0, x)} h(u) P^{n}(x, d u) .
$$

Clearly

$$
\left(P^{n} h\right)(x)=E_{x}\left\{h\left(X_{n}\right)\right\}
$$

The following proposition will often be used.

Proposition 3.1

Suppose that $h(")$ is monotone on $[0, x]$, then

$$
\begin{aligned}
& \lim _{n \rightarrow \infty} \int_{0}^{x-0} \frac{Q\left(x, d x_{1}\right)}{1-\beta\left(x_{1}\right)} \ldots \int_{0}^{x_{n-2}-0} \frac{Q\left(x_{n-2}, d x_{n-1}\right)}{1-\beta\left(x_{n-1}\right)} \int_{0}^{x_{n-1}-0} h\left(x_{n}\right) Q\left(x_{n-1}, d x_{n}\right) \\
& =[1-\beta(x)] h(0+) .
\end{aligned}
$$

Proof:

Since $X_{n} \rightarrow 0$ (a.e) $P_{x}$, it follows from (3.5) and the monotone convergence theorem that

$$
\lim _{n \rightarrow \infty}\left(P^{n} h\right)(x)=h(0+)
$$

On the other hand, for all $\mathrm{n}=1,2, \ldots$ 


$$
\begin{gathered}
\left(\mathrm{P}^{\mathrm{n}} \mathrm{h}\right)(\mathrm{x})=\int_{0 \leq \mathrm{x}_{\mathrm{n}}<\ldots} \ldots \int_{\mathrm{x}_{1}<\mathrm{x}} \mathrm{h}\left(\mathrm{x}_{\mathrm{n}}\right) \mathrm{P}\left(\mathrm{x}, \mathrm{dx_{1 }}\right) \ldots \mathrm{P}\left(\mathrm{x}_{\mathrm{n}-1}, \mathrm{dx} \mathrm{x}_{\mathrm{n}}\right) \\
=\frac{1}{1-\beta(\mathrm{x})} \int_{0}^{\mathrm{x}-0} \frac{\mathrm{Q}\left(\mathrm{x}, \mathrm{dx} \mathrm{x}_{1}\right)}{1-\beta\left(\mathrm{x}_{1}\right)} \ldots \int_{0}^{\mathrm{x}_{\mathrm{n}-\mathrm{-}^{-0}}} \frac{\mathrm{Q}\left(\mathrm{x}_{\mathrm{n}-2}, \mathrm{dx} \mathrm{x}_{\mathrm{n}-1}\right)}{1-\beta\left(\mathrm{x}_{\mathrm{n}-1}\right)} \int_{0}^{\mathrm{x}_{\mathrm{n}-1}-0} \mathrm{~h}\left(\mathrm{x}_{\mathrm{n}}\right) \mathrm{Q}\left(\mathrm{x}_{\mathrm{n}-1}, \mathrm{dx_{n }}\right)
\end{gathered}
$$

By letting $\mathrm{n} \rightarrow \infty$, the assertion follows.

An estimate of the rate of convergence in (3.7) is given by the following:

\section{Proposition 3.2}

Let $h(\cdot)$ be non-decreasing on $[0, x]$, then

$$
0 \leq\left(\mathrm{P}^{\mathrm{n}} \mathrm{h}\right)(\mathrm{x})-\mathrm{h}(0+) \leq \int_{[0, \mathrm{x})} \exp \left(-\mathrm{nP}\left(\mathrm{x}, \mathrm{B}_{\mathrm{u}}^{\prime}\right) \operatorname{dh}(\mathrm{u})\right.
$$

Proof:

We clearly have

$$
E_{x}\left\{h\left(X_{n}\right)\right\}=h(0+)+\int_{[0, x)}\left[1-P^{n}\left(x, B_{u}^{\prime}\right)\right] d h(u) \leq h(0+)+\int_{[0, x)}\left[1-P\left(x, B_{u}^{\prime}\right)\right]^{n} d h(u) .
$$

Now, taking into account (2.5) (the same inequality holds for $\mathrm{P}^{\mathrm{n}}$ ), we have

$$
E_{x}\left\{h\left(X_{n}\right)\right\}-h(0+) \leq \int_{[0, x)}\left[1-P\left(x, B_{u}^{\prime}\right)\right]^{n} d h(u)
$$

which proves (3.9).

\section{SOJOURN TIMES OF $\left\{\zeta_{n}\right\}_{0}^{\infty}$}

Set

$$
\mathrm{T}_{0}=\tau_{0} \text { and } \mathrm{T}_{\mathrm{n}}=\tau_{\mathrm{n}}-\tau_{\mathrm{n}-1} \text { for } \mathrm{n}=1,2, \ldots
$$


This section is concerned with some distributional properties of $\left\{T_{n}\right\}_{0}^{\infty}$. First, it is easy to verify that

$$
\mathrm{P}_{\mathrm{x}}\left\{\mathrm{T}_{0}=\mathrm{k}\right\}=(\beta(\mathrm{x}))^{\mathrm{k}}\{1-\beta(\mathrm{x})\} \mathrm{k}=0,1, \ldots .
$$

The following proposition gives all the marginal distributions of $\left\{\mathrm{T}_{\mathrm{n}}\right\}_{0}^{\infty}$.

\section{Proposition 4.1}

For all $\mathrm{x}>0$ and $\mathrm{n}=1,2, \ldots$,

$$
\begin{aligned}
& \mathrm{P}_{\mathrm{x}}\left\{\mathrm{T}_{0}=\mathrm{i}_{0}, \ldots, \mathrm{T}_{\mathrm{n}}=\mathrm{i}_{\mathrm{n}}\right\} \\
& =(\beta(\mathrm{x})){ }^{\mathrm{i}_{0}} \int_{0 \leq \mathrm{x}_{\mathrm{n}}<\ldots<\mathrm{x}_{1}<\mathrm{x}} \ldots \int\left\{\left(\mathrm{x}_{\mathrm{n}}\right)\right\} \prod_{\mathrm{k}=1}^{\mathrm{n}}\left(\beta\left(\mathrm{x}_{\mathrm{k}}\right)\right)^{\mathrm{i}^{-1}} \mathrm{Q}\left(\mathrm{x}_{\mathrm{k}-1}, \mathrm{dx} \mathrm{x}_{\mathrm{k}}\right),
\end{aligned}
$$

where $i_{k}=1,2, \ldots$ for $k=1,2, \ldots$ and $x_{0}=x$.

Proof:

After some straightforward calculations we obtain:

$$
\begin{aligned}
& P_{x}\left\{T_{0}=i_{0}, \ldots, T_{n}=i_{n}\right\} \\
& =(\beta(x)) \int_{0}^{i_{0}} P_{x_{1}}^{x-0}\left\{T_{0}=i_{1}-1, T_{1}=i_{2}, \ldots T_{n-1}=i_{n}\right\} Q\left(x, d x_{1}\right) \\
& =(\beta(x)){ }^{i_{0}} Q P_{x}\left\{T_{0}=i_{1}-1, T_{1}=i_{2}, \ldots T_{n-1}=i_{n}\right\} .
\end{aligned}
$$

From this recursive formula it follows at once that 


$$
\begin{aligned}
& P_{x}\left\{t_{0}=i_{0}, \ldots, T_{n}=i_{n}\right\} \\
& =(\beta(x))^{i_{0}}\left(Q \beta^{i_{1}-1} \ldots Q \beta^{i_{n}-1}(1-\beta)\right)(x) \\
& =(\beta(x)) \int_{0}^{i_{0}}\left(\beta\left(x_{1}\right)\right)^{i_{1}-1} Q\left(x, d x_{1}\right) \ldots \int_{0}^{x_{n-2}-0}\left(\beta\left(x_{n-1}\right)\right)^{i_{n-1}-1} Q\left(x_{n-2}, d x_{n-1}\right) \\
& \int_{0}^{x_{n-1}-0}\left(\beta\left(x_{n}\right)\right)^{i_{n}-1}\left[1-\beta\left(x_{n}\right)\right] Q\left(x_{n-1}, d x_{n}\right),
\end{aligned}
$$

which proves (4.3).

\section{Corollary 4.1}

The r.v. $\mathrm{T}_{0}$ is conditionally independent of $\left\{\mathrm{T}_{\mathrm{n}}\right\}_{1}^{\infty}$ given $\zeta_{0}$.

\section{Proposition 4.2}

For every $\mathrm{n}=1,2 \ldots$, and $\mathrm{i}=1,2, \ldots$,

$$
P_{x}\left\{T_{n}=i\right\}=\left(P^{n} \beta^{i-1}\right)(x)-\left(P^{n} \beta^{i}\right)(x) .
$$

Proof:

From (4.4) we obtain at once that

$$
\begin{aligned}
P_{x}\left\{T_{n}=i\right\}= & \frac{1}{1-\beta(x)} \int_{0}^{x-0} \frac{Q\left(x, d x_{1}\right)}{1-\beta\left(x_{1}\right)} \ldots \int_{0}^{x_{n-2}-0} \frac{Q\left(x_{n-2}, d x_{n-1}\right)}{1-\beta\left(x_{n-1}\right)} \times \\
& \int_{0}^{x_{n-1}-0}\left\{1-\beta\left(x_{n}\right)\right\}\left(\beta\left(x_{n}\right)\right)^{i-1} Q\left(x_{n-1}, d x_{n}\right)
\end{aligned}
$$

This and (3.8) prove the assertion.

We now prove a result which is needed later.

\section{Proposition 4.3}

For all $\mathrm{n}=0,1, \ldots$, 


$$
\lim _{x \rightarrow 0} P_{x}\left\{T_{0}=i_{0}, \ldots, T_{n}=i_{n}\right\}=(1-p)^{n+1} p^{\sum_{0}^{n} i_{k}-n} .
$$

Proof:

From (2.2) and (2.11) and (4.3) we have:

$$
P_{x}\left\{T_{0}=i_{0}, \ldots, T_{n}=i_{n}\right\} \leq\{1-\beta(x)\}^{n+1} p^{\sum_{0}^{n} i_{k}-n} .
$$

On the other hand,

$$
P_{x}\left\{T_{0}=i_{0}, \ldots, T_{n}=i_{n}\right\} \geq(i-p)^{n+1}(\beta(x))^{\sum_{0}^{n} i_{k}-n} .
$$

By letting $x \rightarrow 0$ the assertion follows.

Remark 4.1

Both functions on the right-hand side of (4.8) and (4.9) are monotone.

Proposition 4.4

The process $\left\{\mathrm{T}_{\mathrm{n}}\right\}_{0}^{\infty}$ possesses a monotonicity property.

Proof:

From (4.5) and (4.6) we clearly have:

$$
\begin{aligned}
& P_{x}\left\{T_{n+1} \geq i\right\}=\left(P^{n+1} \beta^{i-1}\right)(x) \\
& =\frac{1}{1-\beta(x)} \int_{0}^{x-0} \frac{Q\left(x, d x_{1}\right)}{1-\beta\left(x_{1}\right)} \ldots \int_{0}^{x_{n-1}-0} \frac{Q\left(x_{n-1}, d x_{n}\right)}{1-\beta\left(x_{n}\right)} \int_{0}^{x_{n}-0}\left(\beta\left(x_{n+1}\right)\right)^{i-1} Q\left(x_{n}, d x_{n+1}\right) \\
& \geq \frac{1}{1-\beta(x)} \int_{0}^{x-0} \frac{Q\left(x, d x_{1}\right)}{1-\beta\left(x_{1}\right)} \ldots \int_{0}^{x_{n-1}-0}\left(\beta\left(x_{n}\right)\right)^{i-1} Q\left(x_{n-1}, d x_{n}\right)=P_{x}\left\{T_{n} \geq i\right\},
\end{aligned}
$$

and the assertion holds. 


\section{SOME ASYMPTOTIC RESULTS}

From (2.11), (3.7) and (4.5) it follows that

$$
\lim _{n \rightarrow \infty} P_{x}\left\{T_{n}=i\right\}=(1-p) p^{i-1}
$$

We want to extend this result by showing that

$$
\left(\mathrm{T}_{\mathrm{n}+1}, \ldots, \mathrm{T}_{\mathrm{n}+\mathrm{k}}\right) \stackrel{\text { d. }}{\rightarrow}\left(\mathrm{T}_{1}^{*}, \ldots, \mathrm{T}_{\mathrm{k}}^{*}\right) \quad \text { as } \mathrm{n} \rightarrow \infty
$$

for all $\mathrm{k}=1,2, \ldots$, where $\left\{\mathrm{T}_{\mathrm{k}}^{*}\right\}_{1}^{\infty}$ is an i.i.d. sequence of r.v's with common distribution (5.1).

Proposition 5.1

For every $\mathrm{k}=1,2, \ldots$ and $\mathrm{x}>0$,

$$
\lim _{n \rightarrow \infty} P_{x}\left\{T_{n+1}=i_{1}, \ldots, T_{n+k}=i_{k}\right\}=\prod_{j=1}^{k}(1-p) p^{i_{j}-1}
$$

Proof:

Write

$$
\begin{aligned}
& P_{x}\left\{T_{n+1}=i_{1}, \ldots, T_{n+k}=i_{k}\right\} \\
& =\sum_{j_{0}=0}^{\infty} \sum_{j_{1}=1}^{\infty} \ldots \sum_{j_{n}=1}^{\infty} P_{x}\left\{T_{0}=j_{0}, \ldots, T_{n}=j_{n}, T_{n+1}=i_{1}, \ldots, T_{n+k}=i_{k}\right\}
\end{aligned}
$$

From this and proposition 4.1, we deduce that 


$$
\begin{aligned}
& \mathrm{P}_{\mathrm{x}}\left\{\mathrm{T}_{\mathrm{n}+1}=\mathrm{i}_{1}, \ldots, \mathrm{T}_{\mathrm{n}+\mathrm{k}}=\mathrm{i}_{\mathrm{k}}\right\} \\
& =\frac{1}{1-\beta(\mathrm{x})}\left\{\int_{0}^{\mathrm{x}-0} \frac{\mathrm{Q}\left(\mathrm{x}, \mathrm{dx_{1 }}\right)}{1-\beta\left(\mathrm{x}_{1}\right)} \cdots \int_{0}^{\mathrm{x}_{\mathrm{n}-1}-0} \frac{\mathrm{Q}\left(\mathrm{x}_{\mathrm{n}-1}, \mathrm{dx} \mathrm{x}_{\mathrm{n}}\right)}{1-\beta\left(\mathrm{x}_{\mathrm{n}}\right)} \times\right. \\
& \left.\int_{0}^{\mathrm{x}_{\mathrm{n}}-0} \mathrm{P}_{\mathrm{x}_{\mathrm{n}+1}}\left\{\mathrm{~T}_{0}=\mathrm{i}_{1}-1, \mathrm{~T}_{1}=\mathrm{i}_{2}, \ldots, \mathrm{T}_{\mathrm{k}-1}=\mathrm{i}_{\mathrm{k}}\right\} \mathrm{Q}\left(\mathrm{x}_{\mathrm{n}}, \mathrm{dx} \mathrm{n}_{\mathrm{n}+1}\right)\right\} \\
& =\mathrm{P}^{\mathrm{n}+1} \mathrm{P}_{\mathrm{x}}\left\{\mathrm{T}_{0}=\mathrm{i}_{1}-1, \mathrm{~T}_{1}=\mathrm{i}_{2}, \ldots, \mathrm{T}_{\mathrm{k}-1}=\mathrm{i}_{\mathrm{k}}\right\} .
\end{aligned}
$$

By letting $n \rightarrow \infty$ and invoking propositions 3.1 and 4.3 we obtain a proof of (5.3).

\section{Remark 5.1}

From this proposition we conclude, roughly speaking, that remote members of $\left\{\mathrm{T}_{\mathrm{n}}\right\}_{0}^{\infty}$ are i.i.d. r.v's. Next, we shall show that the $\left\{\mathrm{T}_{\mathrm{n}}\right\}_{0}^{\infty}$ possess a mixing property.

\section{Proposition 5.2}

For any $\mathrm{n}=0,1, \ldots$, and $\mathrm{k}=1,2, \ldots,\left\{\mathrm{T}_{\mathrm{i}}\right\}_{0}^{\mathrm{n}}$ and $\left\{\mathrm{T}_{\mathrm{j}}^{*}\right\}_{1}^{\mathrm{k}}$ are independent sequences.

Proof:

$$
\begin{aligned}
& P_{x}\left\{T_{0}=i_{0}, \ldots, T_{n}=i_{n}, T_{n+m+1}=j_{1}, \ldots, T_{n+m+k}=j_{k}\right\} \\
& =\sum_{i_{n+1}=1}^{\infty} \ldots \sum_{i_{n+m}=1}^{\infty} P_{x}\left\{T_{0}=i_{0}, \ldots, T_{n+m}=i_{n+m}, T_{n+m+1}=j_{1}, \ldots, T_{n+m+k}=j_{k}\right\}
\end{aligned}
$$

We now invoke (3.8) and proposition 4.1 to obtain, after some straightforward calculations, that 


$$
\begin{aligned}
P_{x}\left\{T_{0}=\right. & \left.i_{0}, \ldots, T_{n}=i_{n}, T_{n+m+1}=j_{1}, \ldots, T_{n+m+k}=j_{k}\right\} \\
& =(\beta(x)){ }^{i_{0}}\left\{\int_{0}^{x-0}\left(\beta\left(x_{1}\right)\right) i_{1}^{i_{1}-1} Q\left(x, d x_{1}\right) \ldots \int_{0}^{x_{n-1}-0}\left\{1-\beta\left(x_{n}\right)\right\}\left(\beta\left(x_{n}\right)\right){ }^{i_{n}-1} \times\right. \\
& \left.P^{m+1} P_{x_{n}}\left\{T_{0}=j_{1}-1, T_{1}=j_{2}, \ldots, T_{k-1}=j_{k}\right\} Q\left(x_{n-1}, d x_{n}\right)\right\} .
\end{aligned}
$$

By letting $\mathrm{m} \rightarrow \infty$ and invoking propositions 3.1 and 4.3 , we conclude that

$$
\begin{gathered}
\lim _{m \rightarrow \infty} P_{x}\left\{T_{0}=i_{0}, \ldots, T_{n}=i_{n}, T_{n+m+1}=j_{1}, \ldots, T_{n+m+k}=j_{k}\right\} \\
=P_{x}\left\{T_{0}=i_{0}, \ldots, T_{n}=i_{n}\right\} \prod_{v=1}^{k}(1-p) p^{i_{v}-1},
\end{gathered}
$$

and the assertion follows.

\section{Corollary 5.1}

The sequence $\left\{\mathrm{T}_{\mathrm{n}}\right\}_{0}^{\infty}$ is $*$-mixing (Blum-Hanson-Koopmans, 1963). This implies that

$$
\bigcap_{n=1}^{\infty} \sigma\left\{T_{n}, T_{n+1}, \ldots\right\}
$$

is a trivial $\sigma$-algebra (Cohn, 1965; Bartfai and Revesz, 1966). Thus, the sequence $\left\{\mathrm{T}_{\mathrm{n}}\right\}_{1}^{\infty}$ obeys the strong law of large numbers, i.e.,

$$
\mathrm{P}_{\mathrm{x}}\left\{\frac{1}{\mathrm{n}} \sum_{\mathrm{i}=1}^{\mathrm{n}} \mathrm{T}_{\mathrm{i}} \rightarrow \frac{1}{1-\mathrm{p}}\right\}=1
$$




\section{CENTRAL LIMIT PROBLEM}

In applications an approximation for the distribution of $\tau_{\mathrm{n}}$ for large $\mathrm{n}$ might be required. Here we give a sufficient condition for

$$
\begin{array}{ll}
\mathrm{Z}_{\mathrm{n}} \stackrel{\mathrm{d} .}{\rightarrow} \mathrm{Z} \sim \mathrm{N}(0,1) \text { under } & \mathrm{P}_{\mathrm{x}}, \text { where } \\
\mathrm{Z}_{\mathrm{n}}=\frac{1}{\mathrm{~b} \sqrt{\mathrm{n}}} \sum_{\mathrm{k}=1}^{\mathrm{n}}\left(\mathrm{T}_{\mathrm{k}}-\mathrm{a}\right), \quad \mathrm{a}=\frac{1}{1-\mathrm{p}}, \quad \mathrm{b}=\mathrm{a} \sqrt{\mathrm{n}} .
\end{array}
$$

Consider

$$
\varphi_{n}(t, x)=E_{x}\left\{e^{t Z_{n}}\right\}
$$

Form (4.3) we obtain at once that

$$
\begin{aligned}
& \varphi_{n}(t, x)=\sum_{i_{1}=1}^{\infty} \ldots \sum_{i_{n}=1}^{\infty} \exp \left\{t \sum_{k=1}^{n} \frac{i_{k}-a}{b \sqrt{n}}\right\} P_{x}\left\{T_{1}=i_{1}, \ldots, T_{n}=i_{n}\right\} \\
& =\frac{1}{1-\beta(x)} \int \ldots \int\left\{\prod_{\substack{0 \\
0}<\ldots<x_{1}<x}^{n}\left(\frac{\exp \left\{\frac{t}{\sqrt{n p}}\right\}}{1-\exp \left\{t \frac{1-p}{\sqrt{n p}}\right\} \beta\left(x_{k}\right)}\right)\right\} Q\left(x, d x_{1}\right) \ldots Q\left(x_{n-1}, d x_{n}\right) \text {. }
\end{aligned}
$$

We now prove the following:

\section{Proposition 6.1}

For all $\mathrm{x} \geq 0$

$$
\overline{\lim } \varphi_{n}(t, x) \leq e^{\frac{1}{2} t^{2}} .
$$

Proof:

Write (6.2) as follows: 


$$
\varphi_{\mathrm{n}}(\mathrm{t}, \mathrm{x})=\frac{1}{1-\beta(\mathrm{x})}\left\{\int_{0}^{\mathrm{x}-0} \frac{\mathrm{Q}\left(\mathrm{x}, \mathrm{dx}_{1}\right)}{1-\beta\left(\mathrm{x}_{1}\right)} \ldots \int_{0}^{\mathrm{x}_{\mathrm{n}-2^{-}-0}} \frac{\mathrm{Q}\left(\mathrm{x}_{\mathrm{n}-2}, \mathrm{dx}_{\mathrm{n}-1}\right)}{1-\beta\left(\mathrm{x}_{\mathrm{n}-1}\right)} \times\right.
$$

$$
\left.\int_{0}^{x_{n-1}-0} \prod_{k=1}^{n} \frac{1-\beta\left(x_{k}\right)}{\exp \left(\frac{t p}{\sqrt{n p}}\right)-\left[\exp \left(\frac{t}{\sqrt{n p}}\right)\right] \beta\left(x_{k}\right)} Q\left(x_{n-1}, d_{n}\right)\right\}
$$

It is not difficult to verify that

$$
\sup _{u} \frac{1-\beta(u)}{\exp \left(\frac{t p}{\sqrt{n p}}\right)-\exp \left(\frac{t}{\sqrt{n p}}\right) \beta(u)} \leq \frac{1-p}{\exp \left(\frac{t p}{\sqrt{n p}}\right)-\exp \left(\frac{t p}{\sqrt{n p}}\right) p}
$$

and that

$$
\exp \left(\frac{\mathrm{tp}}{\sqrt{\mathrm{np}}}\right)-\exp \left(\frac{\mathrm{t}}{\sqrt{\mathrm{np}}} \mathrm{p}\right)^{-1}=\left(1-\frac{\mathrm{t}^{2}}{2 \mathrm{n}}+\mathrm{o}\left(\frac{1}{\mathrm{n}}\right)\right)^{-1}
$$

as $n \rightarrow \infty$. From this and (6.4) we have

$$
\varphi_{n}(t, x) \leq\left(1-\frac{t^{2}}{2 n}+o\left(\frac{1}{n}\right)\right)^{-n}
$$

By letting $n \rightarrow \infty$ the assertion follows.

\section{Proposition 6.2}

Suppose that for every sequence $\left\{\mathrm{x}_{\mathrm{k}}\right\}_{1}^{\infty}$ such that $\mathrm{x}_{\mathrm{k}} \downarrow 0$

$$
\lim _{n \rightarrow \infty} \frac{1}{\sqrt{n}} \sum_{k=1}^{n}\left[p-\beta\left(x_{k}\right)\right]=0
$$

then

$$
\lim _{n \rightarrow \infty} \varphi_{n}(t, x)=e^{\frac{1}{2} t^{2}} .
$$

Proof: 
As $\mathrm{n} \rightarrow \infty$ we have that

$$
\begin{aligned}
& \prod_{\mathrm{k}=1}^{\mathrm{n}}\left\{\exp \left(\frac{\mathrm{tp}}{\sqrt{\mathrm{np}}}\right)-\exp \left(\frac{\mathrm{t}}{\sqrt{\mathrm{np}}}\right) \beta\left(\mathrm{x}_{\mathrm{k}}\right)\right\} \\
& =\prod_{\mathrm{k}=1}^{\mathrm{n}}\left\{\left(1-\beta\left(\mathrm{x}_{\mathrm{k}}\right)\right)+\frac{\mathrm{t}}{\sqrt{\mathrm{np}}}\left(\mathrm{p}-\beta\left(\mathrm{x}_{\mathrm{k}}\right)\right)+\frac{\mathrm{t}^{2}}{2 \mathrm{np}}\left(\mathrm{p}^{2}-\beta\left(\mathrm{x}_{\mathrm{k}}\right)\right)+O\left(\mathrm{n}^{-\frac{3}{2}}\right)\right.
\end{aligned}
$$

Hence, as $n \rightarrow \infty$

$$
\begin{aligned}
& \prod_{\mathrm{k}=1}^{\mathrm{n}}\left[\frac{1-\beta\left(\mathrm{x}_{\mathrm{k}}\right)}{\exp \left(\frac{\mathrm{tp}}{\sqrt{\mathrm{np}}}\right)-\exp \left(\frac{\mathrm{t}}{\sqrt{\mathrm{np}}}\right) \beta\left(\mathrm{x}_{\mathrm{k}}\right)}\right] \\
& =\exp \left\{-\sum_{\mathrm{k}=1}^{\mathrm{n}} \ln \left[1+\frac{\mathrm{t}}{\sqrt{\mathrm{np}}} \frac{\mathrm{p}-\beta\left(\mathrm{x}_{\mathrm{k}}\right)}{1-\beta\left(\mathrm{x}_{\mathrm{k}}\right)}+\frac{\mathrm{t}^{2}}{2 \mathrm{np}} \frac{\mathrm{p}^{2}-\beta\left(\mathrm{x}_{\mathrm{k}}\right)}{1-\beta\left(\mathrm{x}_{\mathrm{k}}\right)}+\mathrm{O}\left(\mathrm{n}^{-\frac{3}{2}}\right)\right]\right\} \\
& =\exp \left\{-\sum_{\mathrm{k}=1}^{\mathrm{n}}\left[\frac{\mathrm{t}}{\sqrt{\mathrm{np}}} \frac{\mathrm{p}-\beta\left(\mathrm{x}_{\mathrm{k}}\right)}{1-\beta\left(\mathrm{x}_{\mathrm{k}}\right)}+\frac{\mathrm{t}^{2}}{2 \mathrm{np}} \frac{\mathrm{p}^{2}-\beta\left(\mathrm{x}_{\mathrm{k}}\right)}{1-\beta\left(\mathrm{x}_{\mathrm{k}}\right)}\right]+\mathrm{O}\left(\mathrm{n}^{-\frac{3}{2}}\right)\right\} \\
& \geq \exp \left\{-\frac{\mathrm{t}}{1-\mathrm{p}} \frac{1}{\sqrt{\mathrm{np}}} \sum_{\mathrm{k}=1}^{\mathrm{n}} \frac{\mathrm{p}-\beta\left(\mathrm{x}_{\mathrm{k}}\right)}{1-\beta\left(\mathrm{x}_{\mathrm{k}}\right)}-\frac{\mathrm{t}^{2}}{2 \mathrm{np}} \sum_{\mathrm{k}=1}^{\mathrm{n}} \frac{\mathrm{p}^{2}-\beta\left(\mathrm{x}_{\mathrm{k}}\right)}{1-\beta\left(\mathrm{x}_{\mathrm{k}}\right)}+\mathrm{O}\left(\frac{1}{n}\right)\right\}
\end{aligned}
$$

Now, from the conditions of the proposition and a well-known result

$$
\lim _{n \rightarrow \infty} \frac{1}{n p} \sum_{k=1}^{n} \frac{p^{2}-\beta\left(x_{k}\right)}{1-\beta\left(x_{k}\right)}=-1
$$

This, (6.5) and (6.4) then imply that for large $n$

$$
\varphi_{n}(t, x) \geq P^{n} e^{\frac{1}{2} t^{2}}
$$


Therefore,

$$
\underline{\lim } \varphi_{n}(t, x) \geq e^{\frac{1}{2} t^{2}}
$$

for all $\mathrm{x}>0$, which together with (6.3) completes the proof of the proposition.

\section{ACKNOWLEDGEMENT}

A personal communication from Dr. Herman Rost considerably improved this paper. The author gratefully acknowledges his help.

\section{REFERENCES}

[1] Bartfai, P. and Revesz, P. (1967). On a zero one law. Z. Wahrsch. verw. Gebiete 7,43-47.

[2] Blum, J.R., Hanson, D.L. and Koopmans, L.H. (1963). On the strong law of large numbers for a class of stochastic processes. Z. Wahrsch. verw. Gebiete 2, 1-11.

[3] Cohn, H. (1965). On a class of dependent random variables. Rev. Roum. Math. Pures et Appl. 10, 1593-1606.

[4] Daley, D.J. (1968). Stochastically monotone Markov chains. Z. Wahrsch. verw. Gebiete 10, 305-317.

[5] Todorovic, P., Woolhiser, D.A. and Renard, K.G. (1987). Mathematical model for evaluation of the effect of soil erosion on soil productivity. Hydrol. Proc. 1, 181-198.

[6] Todorovic, P. and Gani, J. (1987). Modeling the effect of erosion on crop production. J. Appl. Prob. 24, 787 - 797. 


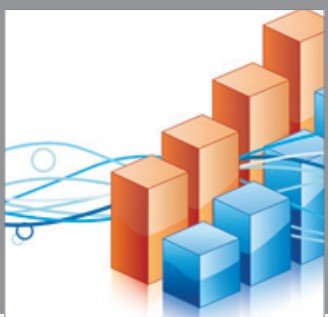

Advances in

Operations Research

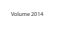

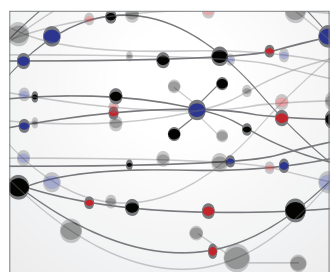

\section{The Scientific} World Journal
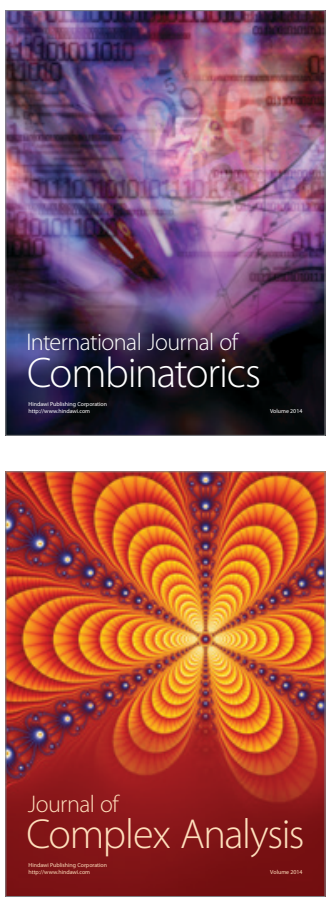

International Journal of

Mathematics and

Mathematical

Sciences
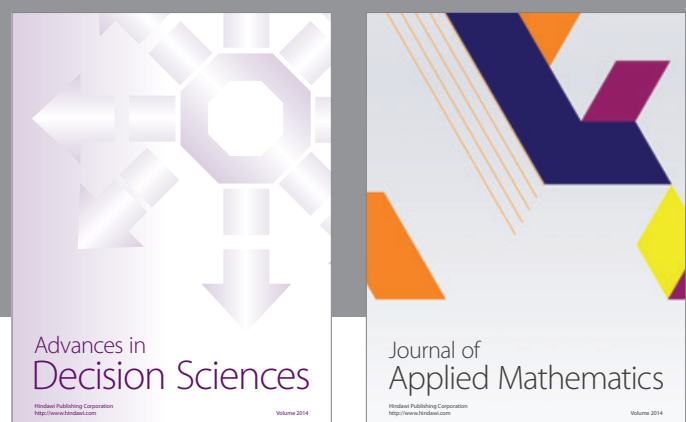

Journal of

Applied Mathematics
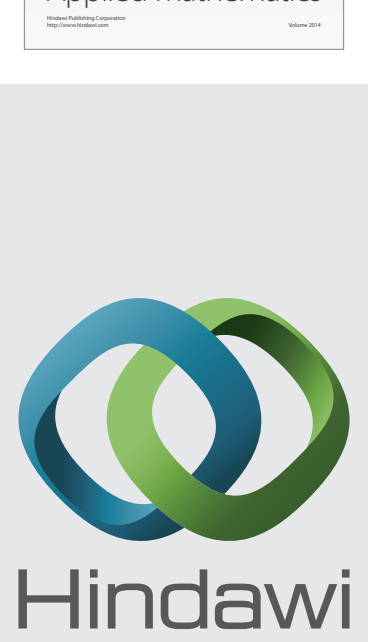

Submit your manuscripts at http://www.hindawi.com
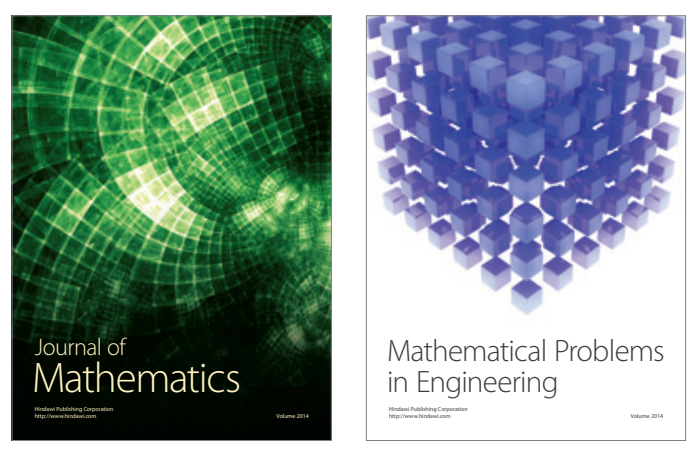

Mathematical Problems in Engineering
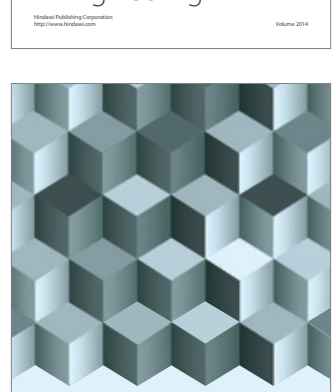

Journal of

Function Spaces
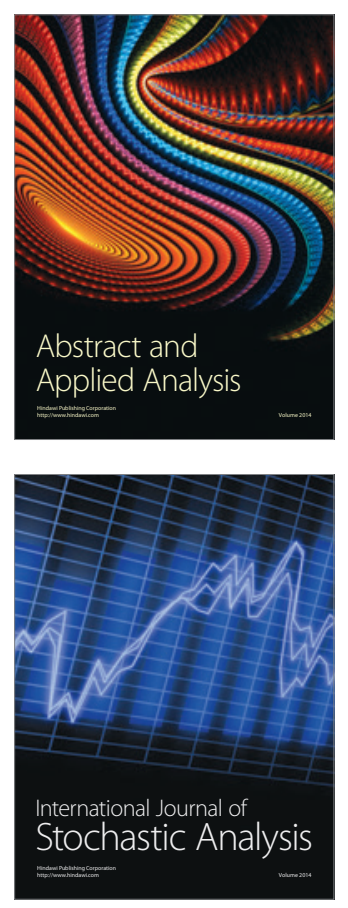

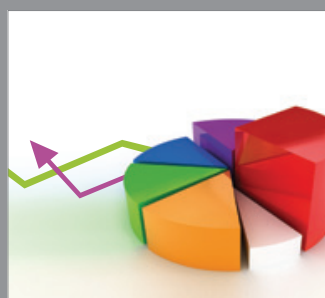

ournal of

Probability and Statistics

Promensencen
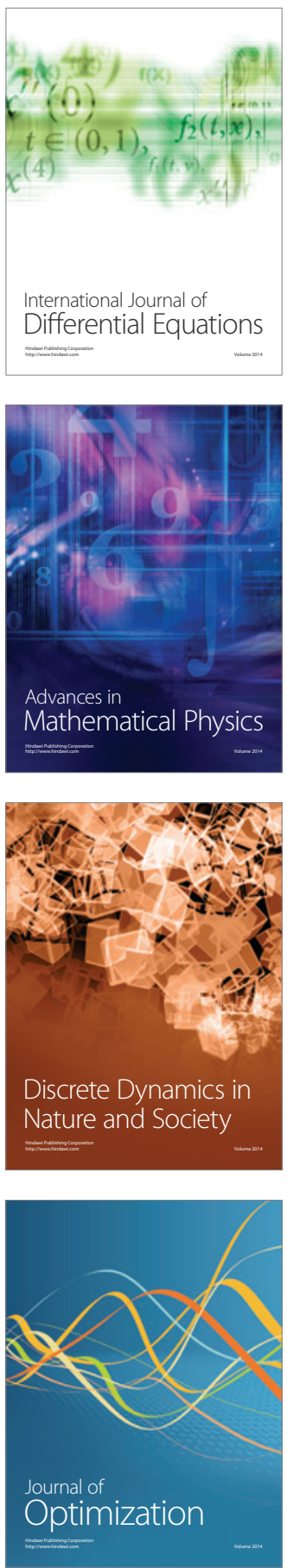